\title{
Satiation or availability? Effects of attention, memory, and imagery on the perception of ambiguous figures
}

\author{
KRISTA L. HORLITZ and ANN O'LEARY \\ Rutgers University, New Brunswick, New Jersey
}

\begin{abstract}
The prolonged-inspection technique has been used to demonstrate effects of satiation on the perception of ambiguous figures. We propose that the inspection phase, in which subjects view an unambiguous version of the stimulus prior to observing the ambiguous figure, does not create neural fatigue but rather provides a context in which the alternative percept is apprehended and gains perceptual strength through processes such as imagination or memory. The consequent availability of the alternative organization drives the perceptual phenomena that have been thought to reflect satiation. In Experiment 1, we demonstrated that (1) preexperimental exposure to the target figures and (2) allocation of attention to the inspection figures were both necessary in order to obtain results similar to those predicted by the satiation model. In Experiment 2, we obtained similar results, finding that effects of prior inspection were greater the greater the amount and availability of information regarding the alternative percept during the inspection phase. Subjects who generated visual images of the noninspected alternative during inspection yielded results comparable to those from subjects to whom both versions were presented visually.
\end{abstract}

Ambiguous figures can give rise to more than one percept. To account for the shifts from one percept to another, Koehler (1940) proposed that neural fatigue resulting from prolonged inspection of one alternative causes the other to arise. As the figure is viewed, fatigue (satiation) develops in response to both alternatives, and the rate of reversal increases. This proposal has generated numerous investigations (e.g., Babich \& Standing, 1981; Carlson, 1953; Cohen, 1959; Hochberg, 1950; Olson \& Orbach, 1966; Orbach, Erlich, \& Heath, 1963; Orbach, Erlich, \& Vainstein, 1963; Spitz \& Lipman, 1962; Toppino \& Long, 1987; Virsu, 1975), and the idea that satiation or neural fatigue is involved in perceptual reversal is generally accepted. Alternative views of reversal, with an emphasis on cognitive variables, have received attention in recent years and will be discussed below (Girgus, Rock, \& Egatz, 1977; Hochberg, 1970; Horlitz, 1988, 1990; Peterson, 1986; Peterson \& Hochberg, 1983; Reisberg \& O’Shaughnessy, 1984; Rock \& Mitchner, 1992; Tsal \& Kolbert, 1985).

The present study was directed at examining cognitive parameters that may have influenced subjects' performance in the classic satiation studies. It was hypothesized that particular settings of these parameters produced re-

This research was supported by the Rutgers University Graduate School of Arts and Sciences. This article is based on K.L.H.'s doctoral dissertation research. The authors wish to thank Irv Rock, Arlene WalkerAndrews, and Hans Wallach for helpful discussions, and Hank Liebeskind and Jim Schubert for technical assistance. Address correspondence to K. L. Horlitz, NASA-Ames Research Center, MS 262-2, Moffett Field, CA 94035 (e-mail: Krista_Horlitz@qmgate.arc.nasa.gov) or A. O'Leary, Department of Psychology, Busch Campus, Rutgers University, New Brunswick, NJ 08903 (e-mail: Oleary@zodiac. rutgers.edu). sults that appeared to fit the satiation model. Specifically, it is our claim that prolonged inspection of an unambiguous figure does not produce fatigue of receptors or visual "channels" but rather provides a period in which the alternative becomes available, and, in turn, that availability influences the perception of the ambiguous figure.

Hochberg (1950) conducted the first formal investigation of satiation as a determinant of figural reversals. It was on the basis of this study that critical questions about the conditions that create satiation, as well as whether or not the satiation model was an adequate description of the data, were posed. In addition to the theoretical issues, a standard methodology emerged. This methodology will be referred to here as the prolonged-inspection technique, which has been used in most experiments on the satiation effect: the subject views an unambiguous version for a period of time (the inspection phase, which is intended to produce satiation), following which the ambiguous figure is presented (the test phase, which is intended to measure the degree of satiation induced). During the test phase, the subject reports the initial perception and, in some studies, each reversal thereafter. Hochberg (1950) introduced the duration of each percept as an indicator of satiation; Carlson (1953) introduced the initial perception measure; and Orbach and colleagues conducted a series of studies in which they examined the time course of satiation by utilizing reversal number as a dependent measure (Olson \& Orbach, 1966; Orbach, Erlich, \& Heath, 1963; Orbach, Erlich, \& Vainstein, 1963). Results that have been taken to support the satiation hypothesis include: (1) greater likelihood of initial perception of the noninspected alternative (e.g., Carlson, 1953; Virsu, 1975); (2) longer total duration of the noninspected 
alternative (e.g., Hochberg, 1950; von Grunau, Wiggin, \& Reed, 1984); and (3) greater frequency of reversals (e.g., Babich \& Standing, 1981; Cohen, 1959; Olson \& Orbach, 1966; Orbach, Erlich, \& Heath, 1963; Orbach, Erlich, \& Vainstein, 1963; Toppino \& Long, 1987), relative to a control condition.

\section{Issues Concerning the Method of the Satiation Studies}

These studies have three features in common. Each feature and its consequences, and how they were addressed in the present study, will be discussed in turn.

1. In the satiation studies, subjects were informed of the reversibility of the target figures and were given experience in reversing them prior to the experimental trials. In fact, the results reported in the satiation studies are in marked contrast to those obtained when subjects do not have information regarding the reversibility of the figure. Epstein and Rock (1960) and Leeper (1935) demonstrated that inspection of an unambiguous version of the figure, immediately prior to exposure to the ambiguous figure, led to the perception of the inspected alternative. Systematic demonstrations of subject awareness of the reversibility of test figures have been provided by Girgus et al. (1977), Horlitz (1988), and Rock and Mitchner (1992). ${ }^{1}$

Girgus et al. (1977) demonstrated that subjects uninformed about the reversible nature of ambiguous figures prior to testing showed greater time to the first reversal and greater likelihood of never reversing the figure in the time given. The method, which differed from the standard prolonged-inspection technique, bears mentioning. The subjects viewed a series of figures, some of which were reversible and others of which were simple drawings that could elicit more than one description. The subjects were prepared to view figures that could be described in more than one way, but the experimenter did not allude to the concept of reversibility. Throughout the viewing period, the experimenter tapped a pencil at 5-sec intervals. Upon this signal, the subjects reported what they saw. A number of experiments, each examining a different test duration, were conducted with Rubin's vase-face and the hallway-pyramid figures. After $1 \mathrm{~min}, 50 \%$ of the naive subjects reversed the figures, and after $3 \mathrm{~min}$, $65 \%$ reversed them. When these subjects were informed (made aware of reversibility and shown the alternatives of the figures) and tested with the same technique, $100 \%$ reversed the figures within $30 \mathrm{sec}$. These results were replicated by Rock and Mitchner (1992), who used a different procedure that relied on the subjective description of the figures by the subjects during a 30-sec test phase. The results from these studies suggest that knowledge of reversibility and possibly the memories of the alternatives that were created when the subjects were presented with drawings representing both alternatives of the figure may have played a role in determining the onset of reversal. It is not clear which of these factors, or whether both of them in concert, determined Girgus et al. 's and Rock and Mitchner's results.
Horlitz (1988) used a prolonged-inspection technique in which the inspection figure was followed by a $1-\mathrm{sec}$ presentation of the ambiguous figure. The critical difference between Horlitz's study and the two studies just described is that in the latter studies there was no inspection phase. Thus Horlitz permitted the first investigation of the relationship between the knowledge factor and the inspection phase in the perception of ambiguous figures.

There were two groups of subjects: one was informed about reversibility, had experience with both alternatives, and had practiced reversing the figures prior to the test. The other group was uninformed about reversibility, did not have experience with both alternatives, and had not had practice in reversing the figures prior to the test. The uninformed subjects were told that they were in an experiment on form perception and that their task was to name each figure that was presented. The prediction was that the uninformed subjects should see the ambiguous test figure as the same as the inspection figure. In contrast, the informed group should report a greater proportion of different responses. A reversible figure from each of the following three categories was used: perspective, figure-ground, and content reversal. Across two experiments, there was a significant difference between groups for the content and figure-ground reversal patterns, and the perspective-reversal figure approached significance $(p=.09)$.

The difference between the informed and uninformed groups suggested the importance of having preexperimental experience with the target ambiguous figures and their alternatives, and knowing the figures were reversible. When subjects were not informed in this manner, they exhibited a past-experience effect. Results from this study suggested that different mechanisms were engaged during the inspection phase, depending on the subject's access to information.

Horlitz's (1988) study suggests that being "informed" may serve to change how the inspection figure is processed, which in turn may influence the subsequent perception of the ambiguous figure. It is plausible that for an informed subject, under the prolonged-inspection method, the alternate organization is not inactive during the inspection phase. On the contrary, information regarding the alternate percept may be available through memory retrieval. This availability could create a situation in which either of the two representations could influence the perception of the ambiguous figure-the figure perceived during the inspection phase and its alternative. Horlitz suggested that the results obtained in the classic satiation studies could be due, in part, to the information that subjects have prior to the test and its availability during the inspection phase. In this study, we isolated these variables.

2. In the satiation studies cited above, a repeated measures design was employed, in which subjects participated in multiple trials with the same figure. This may have permitted trial-to-trial learning. We controlled for this in the present study by using different figures on each trial. 
3. In the satiation studies, measures were not taken to eliminate the effect of demand characteristics. One can infer that subjects hypothesized that the experimenters were interested in figural reversals. If the experimental requirements suggest that reversals are expected and desired as the critical measure, artifactual results may emerge. Indeed, that a subject can control his/her perception of an ambiguous figure when instructed to do so has been demonstrated in experiments by Fluegal (1912), Peterson and Hochberg (1983), and Peterson (1986). This work suggests that subjects are able to perceptually maintain a given organization when instructed to "hold" the percept. In addition, Liebert \& Burk (1985) have demonstrated that perceptual reversals can be controlled voluntarily. The mechanism by which a subject "holds" or maintains a given organization of a reversible figure and the mechanism by which a subject reverses an ambiguous figure may be different, although the aforementioned research suggests that both of these processes can be affected by a subject's intentions. Just how intentions become manifest in perceptual end products-that is, what actions are required by the cognitive system to fulfill intentions-is not clear. Peterson (1986) and Peterson and Hochberg (1989) speculate, and present some interesting evidence, that the perception of form may be the end result of a piecemeal process and thus the order in which pieces of information are obtained (intention affecting order) may influence the direction that the percept takes. In the present study, we were not directly concerned with this issue. However, the results of the present study may assist us in further addressing how intentions influence perception.

In research regarding variables that influence the perception of ambiguous figures, efforts must be made to reduce experimental demand. We employed measures to control demand characteristics by embedding the prolongedinspection task within a cover task to be described in the Method section in Experiment 1. Postexperimental interviews with the subjects indicated that the cover was effective. That is, the subjects were not aware that we were examining figural reversals per se.

\section{Ambiguous Figures and Attention}

The idea that the perception of ambiguous figures can be influenced by a "fluctuation of attention" goes back to Fluegel (1912). This work suggested that the direction of attention determined the apparent organization of an ambiguous figure. Tsal and Kolbert (1985) presented evidence that attention is distributed maximally to an area that supports a particular interpretation of a contentreversal figure such as the duck-rabbit. Reisberg and O'Shaughnessy (1984) found increased time to the first reversal and a reduction in the rate of reversal of ambiguous figures when subjects divided attention between the ambiguous figure and a secondary task. Reisberg and O'Shaughnessy suggest that each act of reconstrual required attentional capacity, some of which was being drained away by the secondary task. The results of all these studies, taken together, suggest that reversals are the end result of a dynamic, active process that requires attention.

In the present study, we examined what effect the allocation of attention to an unambiguous inspection figure has on the subsequent perception of the ambiguous test figure. Although attention should be unnecessary for satiation to occur, as suggested by the following quote- " $\mathrm{Fa}$ tigue theories imply that the multiple neural channels are passive, data-driven processes that operate automatically. ... They are triggered by appropriate retinal stimulation, and require little or no attentional capacity" (Toppino \& Long, 1987, p. 38)-it might well be a necessary prerequisite for cognitive effects to emerge. Focal attention, as conceptualized here, provides the setting in which critical processes emerge, processes that alter the nature of the prolonged inspection experience.

\section{Attention and the Prolonged-Inspection Technique}

We proposed that the prolonged-inspection technique, as used in the satiation studies, provides a subject with an opportunity to prepare for the stimulus that is to follow. This preparation occurs through the apprehension of the alternative version during the inspection phase. A subject, under conditions of focal attention, may undergo a high-level analysis of the figure, resulting in the accessing of multiple memories. If this information becomes available in short-term memory during the inspection phase, the alternate version may become increasingly apparent, thus leading to the increased perceived ambiguity of the inspection stimulus. We predicted that this should increase the likelihood that the ambiguous figure could be perceived in a manner congruent either with the inspection stimulus or with the alternative. The relative strength of these two options may become manifest in the perceived instability (i.e., reversal rate) of the ambiguous figure. The experiments reported here were designed to explore an alternative interpretation of the satiation results that are produced when the prolonged-inspection technique is used. We reduced demand characteristics and other procedural variables that might add to the effect that has been called satiation. We did this in an attempt to examine the influence of the present variables independently.

Experiment 1 was designed to test the effects of two factors-experience with the test figures and the allocation of attention to the inspection figure-on the perception of the test figure. The manipulation of attention to the unambiguous stimulus was used to determine whether the effect of the inspection period was passive and simply due to the stimulation of the system by the figure, or whether the stimulation of the system paired with a higher level process leads to a pattern of results that may appear to be due to satiation. We hypothesized that only under conditions of focal attention would subjects be likely to activate and utilize critical memories during the inspection phase.

In Experiment 2, the amount and availability of information regarding the noninspected alternative was systematically varied to test the hypothesis that greater avail- 
ability would be associated with stronger resemblance of results to those predicted by a satiation model (greater proportion of the responses that are opposite to the inspection stimulus, longer duration of the noninspected alternative, and greater number of reversals).

In both experiments, a learning phase was provided to familiarize subjects with the stimuli. During this learning phase, some groups received experience with the target figures to be used in the critical experiment and other groups received experience with figures that were similar to the target figures. Next, a practice phase acquainted subjects with the experimental procedure. This was done to ensure that the experimental phase ran smoothly and that the subjects understood the sequence of events that was to follow. The target test figures were not used during this phase. During the experimental phase, inspection and ambiguous figure pairs were interspersed with distractor figures. A cover task, which will be discussed in the Method section of Experiment 1, was used to reduce experimental demand.

\section{EXPERIMENT 1}

In Experiment 1, undergraduate subjects were assigned randomly to one of three conditions. Those in the Attention/Familiar condition were given experience with the target figures during the learning phase and were instructed to provide focal attention to the inspection figures during the experimental phase. The Inattention/Familiar group also received experience with the target figures during the learning phase, but they did not attend to the inspection figures during the experimental phase. This condition was created by superimposing two flashing lights that furnished a distracting two-dot task in the center of the inspection figures. The subjects were told to focus their attention on these flashing lights and to count the times both lights were on simultaneously. The Attention/ Unfamiliar subjects did not see the target figures during the learning phase, but they did see a number of reversible figure-ground patterns that were similar to the target figures. They were instructed to provide focal attention to the inspection figures during the experimental phase. The subjects in each group allocated focal attention to the ambiguous test figures.

We hypothesized that subjects who had attended to the inspection figures and who had had prior experience with those figures would exhibit a tendency to see the opposite version of the ambiguous figure first, would prefer the noninspected version, and would exhibit a faster rate of reversal, in comparison with subjects who also had prior experience with the figures but had not provided focal attention. The subjects in the Attention/Unfamiliar group were expected to perform similarly to those in the Inattention/Familiar group in terms of the number of reversals, because in both of these conditions availability of the alternative version was minimal. The Attention/ Unfamiliar subjects were expected to exhibit a pastexperience effect, when measured by the initial percep- tion of the ambiguous figures, reflected in a tendency to perceive the inspected form of the figure, whereas the subjects in the Inattention/Familiar group were expected not to show a tendency to prefer one or the other interpretation, because of the shallow level of processing provided to the inspection stimulus.

\section{Method}

\section{Subjects}

Twenty-nine females and 25 males, recruited from the general subject pool at Rutgers University, participated as part of a course requirement. Nine subjects were eliminated from the study because of failure to reverse one or more of the figures during a screening process, and 1 subject was eliminated because of equipment failure. Ultimately there were 15 subjects in the Inattention/Familiar and the Attention/Unfamiliar groups and 14 in the Attention/Familiar group. All had normal or corrected vision.

\section{Stimuli}

The figure-ground reversal patterns displayed in Figure 1 were selected for the study for three reasons. First, they were novel, enabling the experience manipulation. Second, they were generally unbiased (indicated by pilot tests) central-contour reversible patterns, which allowed standardization of how subjects reported reversals of the figures- "left" or "right." This feature was important, because it permitted the experimenter to collect meaningful responses from subjects who were otherwise unfamiliar with the figures that were being used. And third, we opted not to use a colored region for either the left- or the right-side inspection figures, because we reasoned that to use colored figures on some trials would have interfered with the inattention manipulation.

All patterns measured $2.54 \times 2.54 \mathrm{~cm}$ and were viewed from a distance of $56 \mathrm{~cm}$, thus subtending a visual angle of $2^{\circ}$. Six distractor figures that were similar in size and shape to the inspection figures were used. The function of the distractor figures will be made clear in the Procedure section. They can be seen in Figure 2. The flashing dots, which were present in all conditions during the presentation of the inspection and distractor figures, were generated by a Radio Shack color computer and were $1 \mathrm{~mm}$ in diameter. Their placement can be seen in Figure 3. The rate of presentation was 160 dot signals per minute. The two dots were on simultaneously either 55 or 66 times during the $60-\mathrm{sec}$ inspection and distractor trials.

\section{Apparatus}

Goggles were placed on the front of a Scientific-Prototype twochannel tachistoscope, Model 800F, with the left side occluded with black tape. All subjects viewed the stimuli with the right eye, through a hole. Monocular viewing was selected for two reasons: Experience with these figures suggested that figure-ground perception was enhanced when the figures were viewed monocularly; and it was desirable to isolate stimulation to a single eye across the inspection and the test phases to prevent the subjects from viewing the inspection figures with one eye and the test figures with the other eye. If satiation, as a form of receptor or channel fatigue, were the underlying mechanism for the pattern of results obtained in a prolongedinspection experiment, the conditions given by this experiment provided optimal stimulation conditions for its occurrence. Thus, this methodological decision was a safeguard against the criticism that we did not provide the proper arena for satiation to emerge.

A computer monitor with a dim screen was placed within one channel of the tachistoscope in order to present the two-dot task. The distractor, inspection, and ambiguous figure-ground patterns were presented in the other channel. A microphone was mounted on the front of the tachistoscope in order to record the responses of the subjects. The time course of responses was transcribed from 


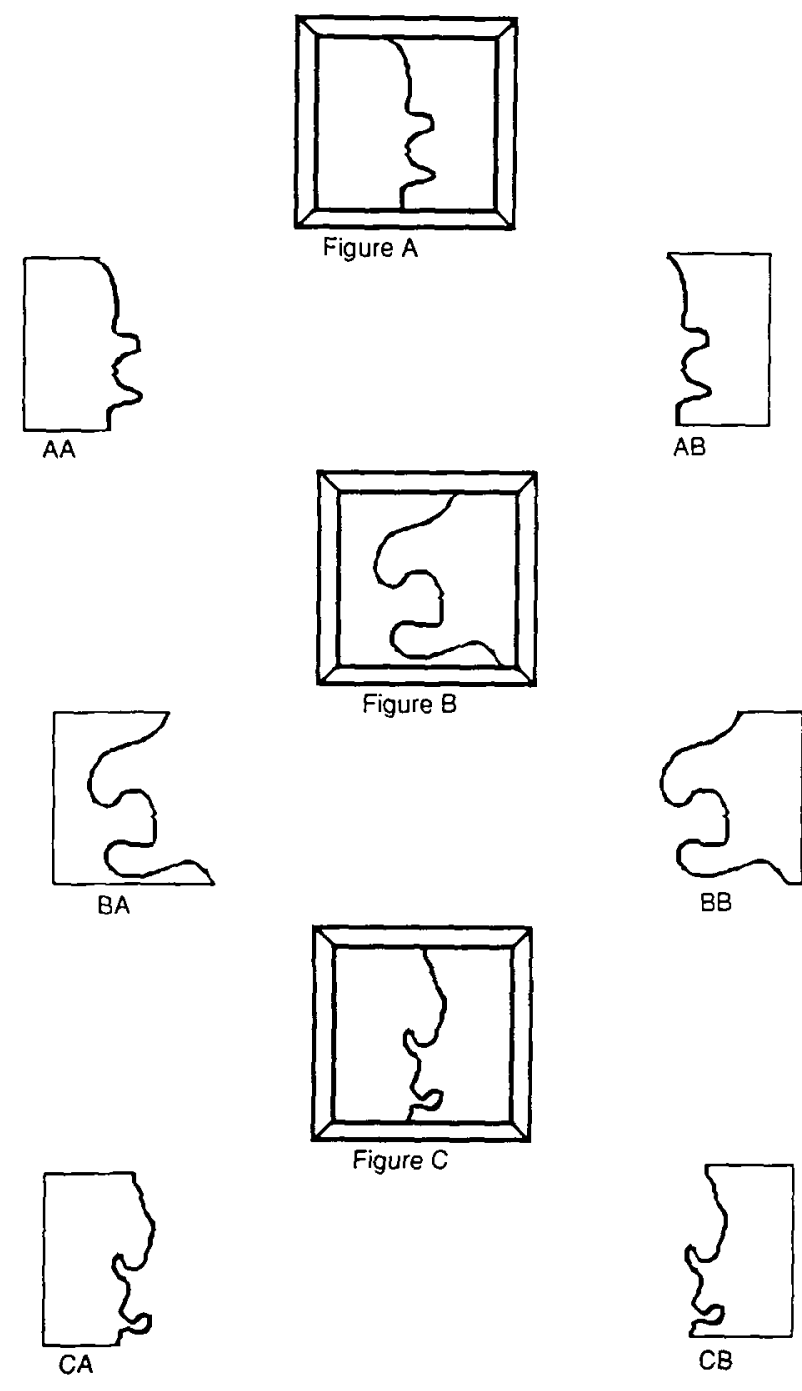

Figure 1. Ambiguous and inspection figures for Experiment 1.

tape to a Commodore computer. This program provided the experimenter with a ticker-tape record of the subjects' responses from the onset to the cessation of the test figures. ${ }^{2}$

\section{Procedure}

The experimental session comprised three phases: learning, practice, and experimental. The experimenter portrayed each phase as a separate experiment. This approach was taken to reduce the possibility that subjects would create a goal state to reverse the figures in the experimental phase. For the treatment of each group at each phase, see Table 1.

Step 1: The learning phase. Two booklets that contained reversible figure-ground patterns were made. Each page of the booklet contained an ambiguous figure-ground pattern on the top and the two alternative interpretations of it at the bottom. If a subject had difficulty perceiving both sides of the reversible figure, it was suggested that looking at the alternatives might help. The subjects in all three groups were instructed to look at the figures until they were able to see that they were reversible. They were told that this was a pilot experiment to investigate whether or not the drawings could elicit more than one interpretation.
The subjects in the Attention/Familiar and Inattention/Familiar groups looked at a booklet that contained both the three figures that would subsequently appear in the test and five other central-contour reversible figure-ground figures. The Attention/Unfamiliar group looked at a booklet with four central-contour reversible figureground figures, none of which were to be test figures. Thus, these subjects were not exposed to the target figures, yet they were provided with experience with similar central-contour ambiguous figures, thereby receiving "general experience" concerning the reversibility of such figures.

Step 2: The practice phase. The purpose of this phase was to ensure that the subjects could smoothly perform the tasks that would be required in the experimental trials. This was accomplished by training them to perform one task, and then to be alerted by an auditory signal from the computer to the onset of a reversible figure. Upon presentation of the figure, the subjects were required to report their initial perception and all reversals. They were told that this cycle would occur recursively.

The practice phase consisted of five trials. During each trial, five arrays, with one to five dots in each array, were presented for $1 \mathrm{sec}$ each. After the five arrays had been presented, a beep indicated that a report of how many dots in all were presented was required. After the report, a reversible figure-ground pattern was presented, and subjects reported their initial perception by saying "left" or "right," and every change thereafter by saying "left" or "right." The target figures were not used in this phase of the study. The nontarget figures that were presented in the learning booklets were used as the reversible figures in this phase. The subjects were told that this was a pilot experiment to examine task switching.

Step 3: The experimental phase. During the experimental phase of the study, a modification of the prolonged-inspection technique was employed for all three groups. The experimental trials (an inspection figure followed by its ambiguous figure pair) were separated by distractor trials. The distractor trials were not followed by ambiguous figures. This addition to the prolonged-inspection technique provided a cover task for the Attention/Familiar and Attention/Unfamiliar groups-namely, one requiring the subject to learn the figures for a recognition test. These subjects were told that the experiment was intended to examine what effect seeing ambiguous figures had on their memory for the figures to be learned. In addition to reducing experimental demand, this technique provided a measure for exploring the effects of the attention manipulation-that is, a recognition test (to be explained in detail later).

The Attention/Familiar and the Attention/Unfamiliar groups viewed a series of figures, each for $60 \mathrm{sec}$, and were instructed to fixate the flashing dots while they spread their attention to the figures. They were informed that the computer would beep occa-
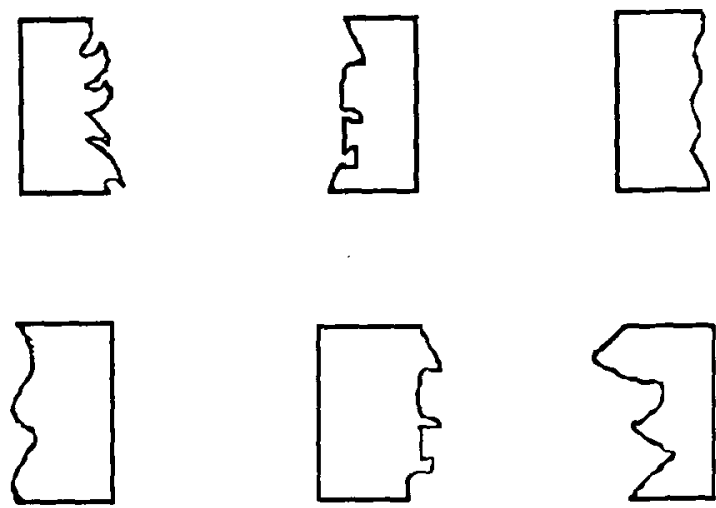

Figure 2. Distractor figures for Experiments 1 and 2. In Experiment 2, half the figures were gray. 


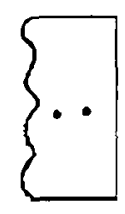

Figure 3. Placement of the two-dot task in Experiment 1.

sionally and that the next trial would be a reversible figure-ground pattern to which they would report their initial perceptions and each reversal.

The Inattention/Familiar group was told that they would participate in a series of 60 -sec trials in which two dots would flash on and off in the middle of the screen. The subjects were instructed to focus their attention on the dots and count the number of times both dots were on simultaneously. They were told that the experimenter was interested in seeing how well people could attend to a difficult visual task when distracting information (the inspection and distractor figures) was present. They too were informed that the computer would beep, following which a reversible-figure trial would occur. During presentation of the reversible figures, the flashing dots were no longer superimposed on the figures.

All three groups were presented with the distractor and inspection figures for $60 \mathrm{sec}$ each and the reversible figure-ground patterns for $10 \mathrm{sec}$. The sequence of trials was as follows: Distractor 1, Distractor 2, Distractor 3, Inspection Figure 1, Reversible Figure-Ground 1, Distractor 4, Distractor 5, Inspection Figure 2, Reversible Figure-Ground 2, Distractor 6, Inspection Figure 3, Reversible Figure-Ground 3. The order of the distractor figures was the same for each subject, but the order of the figure-ground patterns was randomized. We used different numbers of distractor trials between the experimental trials in hopes of reducing subjects' expectations about what type of trial would occur at any point in time.

As an internal control, there were two subgroups in each of the three groups. Subgroup 1 saw Inspection Figures AB, BB, and CA, whereas Subgroup 2 saw AA, BA, and CB (see Figure 1). This ensured that an equal number of left and right inspection figures were presented and that each subject experienced at least one inspection figure that was from the left side of the figure and one that was from the right side of the figure. Thus, each alternative of each of the ambiguous figures was used as an inspection figure. This served to counterbalance any specific inspection figure effects that might have emerged across subjects within the three groups. Subgroup was not intended as a factor of interest. There was the following distribution of subjects per subgroup per group: Attention/ Familiar, 6 in Subgroup 1, 6 in Subgroup 2, 1 subject who received all inspection figures from the right, and 1 subject who received all inspection figures from the left; Attention/Unfamiliar: 7 subjects in Subgroup 1 and 8 subjects in Subgroup 2; Inattention/ Familiar: 6 in Subgroup 1, 7 in Subgroup 2, 1 subject who received inspection figures all from the right, and 1 subject who received inspection figures all from the left.

\section{Recognition Test}

After completing the experiment, all subjects were given a multiple-choice recognition test. The recognition test provided a measure of three things. (1) It assessed the effectiveness of the inattention manipulation, in that if subjects were allocating the majority of their attention to the two-dot task, they should receive a low score on the recognition test. (2) If subjects in the Attention/Familiar and Attention/Unfamiliar groups were behaving according to the task goals established by the experimenter, they should show a relatively high score for the recognition test. (3) We could examine the relationship between the recognition score and the measures of perceptual reversal.

There were six recognition items. For each item, there were three foils that were never shown and one distractor (see Figure 2) that was actually shown during the experiment. The foils were drawn to look similar to the distractors. The subjects were asked to circle one figure from each row (one through six) indicating the figure seen during the experiment. They were told that the figures were not in the order in which they had been presented during the experiment.

After the completion of the recognition test, the subjects were interviewed and debriefed. During the interview, the experimenter ascertained whether or not the subjects had formed the hypothesis that the experiment was ultimately intended to have them reverse or not reverse the figures.

\section{Results}

A number of measures were examined: number of reversals for each group, duration of the inspected version, and the initial percept. Each of these measures will be defined in turn. The summary data can be seen in Table 2 .

\section{Number of Reversals}

It was predicted that the Attention/Familiar subjects should perceive a greater number of reversals than either of the other groups because these subjects were provided with the opportunity to engage in a deep level of processing during the inspection period, which may have entailed the evocation of the alternate figure. We have speculated

Table 1

Treatment of the Groups in Experiment 1

\begin{tabular}{|c|c|c|c|}
\hline \multirow[b]{2}{*}{ Phase } & \multicolumn{3}{|c|}{ Group } \\
\hline & Attention/Familiar & Attention/Unfamiliar & Inattention/Familiar \\
\hline $\begin{array}{l}\text { Learning } \\
\text { Figures }\end{array}$ & target & nontarget & target \\
\hline $\begin{array}{l}\text { Practice } \\
\text { Figures }\end{array}$ & nontarget & nontarget & nontarget \\
\hline $\begin{array}{l}\text { Experimental } \\
\text { Instructions }\end{array}$ & $\begin{array}{l}\text { learn figures, } \\
\text { report reversals }\end{array}$ & $\begin{array}{l}\text { learn figures, } \\
\text { report reversals }\end{array}$ & $\begin{array}{l}\text { two-dot task, } \\
\text { report reversals }\end{array}$ \\
\hline Figures & $\begin{array}{l}\text { target, } \\
\text { Inspection/Ambiguous }\end{array}$ & $\begin{array}{l}\text { target, } \\
\text { Inspection/Ambiguous }\end{array}$ & $\begin{array}{l}\text { target, } \\
\text { Inspection/Ambiguous }\end{array}$ \\
\hline
\end{tabular}

Note-The subjects' purpose in the learning phase was to create critical traces of the target features or not; in the practice phase, to learn the procedure. 
Table 2

Summary Data From Experiment 1

\begin{tabular}{lccc}
\hline & \multicolumn{3}{c}{ Group } \\
\cline { 2 - 4 } \multicolumn{1}{c}{ Dependent Variable } & Attention/Familiar & Inattention/Familiar & Attention/Unfamiliar \\
\hline Mean No. of Reversals & 4.05 & 1.84 & 1.56 \\
Duration (same, in seconds) & 5.56 & 5.26 & 5.93 \\
Initial percept (different) & $43 \%$ & $40 \%$ & $33 \%$ \\
Recognition score & $65 \%$ & $33 \%$ & $73 \%$ \\
Correlation coefficients & & & \\
between reversal number & & .59 & .63 \\
and recognition score & -.25 & .59 & \\
\hline
\end{tabular}

that this evocation makes the trace of both the perceptually given inspection figure and the memory of the alternative available to working memory. It was predicted that the availability of these traces would lead to a greater number of reversals. Furthermore, it was predicted that the Attention/Unfamiliar and Inattention/Familiar groups would not differ significantly from one another, because of the relatively shallow processing of the inspection figures. In the case of the Attention/Unfamiliar group, the subjects did not receive specific experience with the target figures during the learning phase; hence, it was predicted that they would perceive the inspection figures as simple figures that did not "belong" to a more complex ambiguous figure. If this were true, it would be quite likely that during the inspection period these subjects would simply encode the properties of the unambiguous figure. Working memory, as it relates to this task, would contain the single representation of the inspection figure. This cognitive state of affairs was predicted to lead to a relatively lower number of reversals. In a similar vein, subjects in the Inattention/Familiar condition were predicted to have the same reversal rate as the subjects in the Attention/Unfamiliar group. Although the Inattention/ Familiar group was familiar with the figures, they were unable to devote a deep level of processing to the inspection figures because of the two-dot task. Although all subjects in this group claimed that they could perceive the figure that was surrounding the two-dot task, in essence, they were not permitted to think about what they were seeing or to think about what it reminded them of and whether or not they had seen it before and, if so, whether or not it was one of the parts of the ambiguous figures that they had been exposed to in the learning phase. ${ }^{3}$ Thus, it is our claim that it is not simple exposure to the target figures prior to the experimental phase that is critical for a different reversal rate among these groups, but rather the level of processing that is engaged during the inspection phase. The level of processing that draws in the representation of the alternate organization of the ambiguous figure will lead to the greatest number of reversals.

These specific predictions were tested in an analysis of variance (ANOVA) with planned contrasts (Rosenthal \& Rosnow, 1985), assigning the following coefficients to the Attention/Familiar, Attention/Unfamiliar, and Inattention/Familiar groups: $2,-1,-1$, respectively. This analysis yielded a significant result $[F(1,41)=6.64, p=$ $.013]$, indicating that the predictions were supported by these data.

\section{Duration}

This variable was defined as the amount of time the subject saw the inspection form of the ambiguous figure during the $10-\mathrm{sec}$ test period. The mean across the three figures was used as the dependent measure. It was predicted that the Attention/Unfamiliar group would have the longest duration for the inspected alternative (the pastexperience effect), that the Inattention/Familiar group would see each alternative for approximately $50 \%$ of the time because these subjects were not likely to be influenced by the inspection figure, and that the Attention/ Familiar group would perceive the inspected version for the shortest time. The coefficients assigned were 2.0 , and .5 , respectively. This analysis yielded a nonsignificant result $[F(1,41)=.84, p>.10]$. There was no evidence of a past experience or satiation effect when duration of the inspected figure was the measure.

\section{Initial Perception Data}

This variable was defined as whether the first perception of the ambiguous figure was the same as the inspection form, or whether it was different (the alternate version). The percent of different responses of the three initial percepts was the dependent measure. The specific figure effects were not examined.

It was predicted that the Attention/Familiar group should have a higher proportion of different percepts in comparison with the Inattention/Familiar and the Attention/Unfamiliar groups. The evocation and availability of the alternate during the inspection phase should lead to a greater probability that this representation would affect the initial perception of the ambiguous figure. The Attention/Unfamiliar group would tend to see the inspected form (that is, would exhibit a past-experience effect), owing to the representation of the inspection figure alone. The Inattention/Familiar subjects would perceive the two versions with equal likelihood because of the negligible influence of the inspection period. The results from the ANOVA with planned orthogonal contrasts (Attention/ Familiar, 2.0; Inattention/Familiar, -1.5; Attention/ Unfamiliar, -.5$)$ was $F(1,41)=.23, p>.10$. 


\section{Recognition Scores}

All three groups received a recognition test at the end of the experiment. Chance performance on this test would be $25 \%$. Of primary interest was whether or not the twodot task in the Inattention/Familiar group had sufficiently distracted subjects from the background figures. The Inattention/Familiar group did not differ significantly from chance $(25 \%)[t(14)=1.49, p>.10]$. The Attention/Familiar group and the Attention/Unfamiliar group did not differ from one another $[t(27)=.98, p>.10]$; and both groups had significantly greater than chance performance $[t(13)=6.507, p<.001$, and $t(14)=9.388, p<.001$, respectively].

It was predicted that, among subjects in the Inattention/Familiar group, higher recognition scores would be associated with greater signs of perceptual change during the test phase, since those who remembered could be presumed to have more successfully allocated attention to the stimulus despite the distracting task and thus engaged in a relatively deeper analysis. A Spearman rankorder correlation was conducted between the recognition scores and reversal rate for subjects in the Inattention/ Familiar group. The two measures were indeed positively correlated $[r(13)=.59, p<.01]$. This correlation was not significant for the Attention/Familiar group $[r(12)=$ $-.25, p>.10$ ], in part due to the restricted range. However, a positive correlation did emerge in the Attention/ Unfamiliar group $[r(13)=.63, p<.01]$.

\section{Discussion}

In this experiment, we examined whether what has been called satiation requires a subject to provide focal attention to the inspection "satiating" figure or whether it is simply a matter of the presence of the figure on the retina. If the satiation-type effect emerges as the end result of an active process of visual problem solving that engages past experience in the form of memories and not one that is due to the passive fatiguing of neural channels, then focal attention should be a necessary condition for the production of the effect. It was of further interest to examine the role that a subject's specific past experience with the alternatives of the figure-ground patterns might play in conjunction with focal attention to the inspection figure.

The present study provided evidence that both focal attention and familiarity with the target reversible figures increased the rate of reversal. This suggests that there was an increase in the availability of critical information during the inspection phase that was brought to bear on the perception of the ambiguous figure.

The positive correlations that were obtained between the number of reversals and the recognition scores for the Inattention/Familiar and the Attention/Unfamiliar groups can be understood by an analysis of what may be occurring during the inspection phase. Subjects in the Inattention/ Familiar group who received relatively high recognition scores were most likely able to distribute their attention between the two-dot task and the perception and learning of the figures. Because they were able to allocate some measure of resources to these figures, and because they were preexposed to the target figures, it is possible that this activated memories created during the preexperimental learning phase. This process would make available the relevant traces in working memory that might increase the number of reversals.

There was no correlation between the reversal number and recognition scores for the Attention/Familiar group, but there was a positive correlation for the Attention/ Unfamiliar group. Why the difference? The critical distinction between these groups was that the Attention/ Familiar group had had previous experience with the figures and the Attention/Unfamiliar group had not. Was it the case that some subjects in the Attention/Unfamiliar group were engaging in a process that allowed for a higher recognition score and a greater number of reversals? A report from 1 subject in this group is informative. This subject reported that to remember the figures, he engaged in the following mnemonic: During the 60-sec inspection phase, while attempting to learn the figures, he found himself imagining what the other side of the figure looked like. He reasoned that this would help him select the correct figure during the test because he would have two pieces of information instead of one and this would increase his probability of a correct selection. It could well be that this strategy does yield a higher rate of recognition and, furthermore, that such a subject was providing himself with experience of a form that did not exist in the stimulation; it was a synthesis of externally derived and internally generated information. Although this subject did not have specific past experience with the inspection or test figures, because of this process of imagining he had created experience that might aid in perceiving both versions of the reversible figure and hence increase the number of reversals. This report is compatible with the idea that some type of learning occurs during the inspection phase and that either preexisting memories become reinforced or new ones are created. It also sheds light on why completely naive subjects can reverse ambiguous figures if given enough time. If one imagines different vantage points, figure-ground organizations, and possible contents, this can lead to the very first reversal. The correlation between the recognition scores and reversal number, and the reports of some of the subjects, provide an interesting index of depth of processing that suggests not only what might be occurring during the inspection phase but also what the underlying process of reversal might be.

The duration data indicated that, on the average, the three groups saw each alternative for approximately $50 \%$ of the viewing period, and there was no difference in the initial perception data although the Attention/Unfamiliar data were in the predicted direction. These analyses suggested that there was neither a satiation-type effect nor a past-experience effect concerning which side of the reversible pattern was perceptually dominant. These data run counter to the fatigue explanation of previous studies. Although it was predicted that the Attention/Familiar subjects would exhibit a tendency to see the noninspected ver- 
sion first, it could be that the memories that were created during the learning phase were not strong enough to become activated during the inspection phase across all subjects and to the same degree. Recall that subjects learned eight different figures during the learning phase, only three of which were the target test figures. It could be that this reduced the effect of the learning because the subjects experienced interference between the figures, which were all very similar. In the follow-up study reported below, only the target test figures were exposed during the learning phase, to see whether this would create a difference in the initial perception data.

\section{EXPERIMENT 2}

Experiment 2 was designed to explore more fully the effects of cognitive processes, such as memory retrieval and the influence of actively imagining the alternate version of the figure during the inspection phase. Four experimental groups and one control group were used. All subjects in the experimental groups allocated focal attention to the inspection figures. Two groups replicated those used in Experiment 1. Subjects in the Familiar group were given experience with the target figures before the test, and those in the Unfamiliar group had no experience with the target figures but were given experience with four reversible figure-ground patterns similar to the target figures. Group A/B received experience with the target figures before the test, and they saw both inspection figures during the inspection phase, each for half of the inspection period-thus the name $A / B$, because they saw Inspection Figure $A$ and Inspection Figure $B$ for each figure. The Imagery/Unfamiliar group received the same preexperimental treatment as the Unfamiliar group, but this group was instructed to imagine what the other side of the inspection form would look like during the inspection phase. Finally, a No-Inspection group (the control) was given experience with the target figures before the test but did not receive the prolonged-inspection technique. They were simply shown the reversible figureground patterns embedded within the series of distractor figures (the cover task) as were the other groups. The only items missing from the stimulus sequence were the inspection figures.

The hypothesis was that as the availability of information during the inspection phase was increased, the greater would be the reversal rate, and further, that this availability should increase the likelihood that a subject would perceive the ambiguous figure opposite to the inspection figure upon initial presentation and during the duration of the test period.

The availability of information in the five groups was conceptualized in the following way:

$$
\begin{aligned}
\text { No-Inspection } & =\text { Unfamiliar }<\text { Familiar }<\text { A/B } \\
& =\text { Imagery/Unfamiliar. }
\end{aligned}
$$

It was predicted that the Imagery/Unfamiliar group and Group A/B would yield comparable results, showing the greatest signs of perceptual reversal and a greater chance of perceiving the ambiguous figure opposite the inspection form than the other two experimental groups. The No-Inspection group data were of interest for the following reasons: (1) to determine whether the figures were biased; and, (2) to obtain a baseline reversal rate when no inspection figure precedes the ambiguous stimulus. It was predicted that the Unfamiliar group would obtain a reversal rate similar to that for the No-Inspection condition. In summary, we predicted that the No-Inspection and Unfamiliar groups would yield the fewest signs of perceptual change, whereas the A/B and Imagery/Unfamiliar groups would exhibit the most, with the Familiar group showing intermediate effects.

\section{Method}

\section{Subjects}

Ninety subjects from the same subject pool as in Experiment 1 participated. Eight were eliminated from the study because they did not follow instructions, and 2 because they did not perceive the figure-ground patterns as reversible. Ultimately there were 16 subjects per group.

\section{Stimuli}

The figure-ground and inspection figures can be seen in Figure 4 . These figures fulfill the same criteria as those in Experiment 1 and were the same size. Recall that in Experiment 1 shading the figures would have interfered with the inattention manipulation and thus was avoided. Without this consideration in the present experiment, half the figures were shaded gray on the right side and half on the left side. These figures were shaded because it made the figures easier to reverse.

\section{Apparatus}

The apparatus was the same as that in Experiment 1. The CRT was no longer mounted within the tachistoscope since the flashing dots were no longer necessary.

\section{Procedure}

As in Experiment 1, there were three phases: learning, practice, and experimental; the purpose of each phase was the same as in Experiment 1. The subjects in all five groups participated in the learning phase with the subjects in groups Unfamiliar and Imagery/ Unfamiliar seeing four reversible figure-ground patterns that were not the test figures. For treatment of each group at each phase, see Table 3.

The subjects in the other three groups were presented with the four figures that were going to be used in the experiment. The procedure of the learning phase was the same as that in Experiment 1.

All subjects participated in the practice phase, which was the same as in Experiment 1, except that instead of the dot-array counting task, subjects were shown a series of geometric figures that they were asked to learn for future recall. Again, the critical aspect of this phase was to ensure that the subjects learned that when the computer emitted a beep, that indicated the onset of an ambiguous figure. The test figures were not used in this phase.

The critical experimental phase was conducted in a manner similar to that of Experiment 1. The instructions to the subjects were the same except for the instructions given to those in the Imagery/ Unfamiliar group. These subjects were told that they were going to see a series of figures that they must learn. In addition to learning the figures presented, they were instructed to imagine what the missing half of each figure would be and to remember these figures along with the ones presented. At this point, the experimenter demonstrated how to imagine these figures by drawing a dashed 


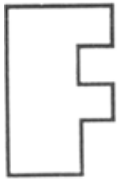

AA

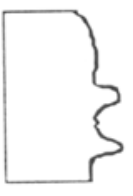

$\mathrm{BA}$

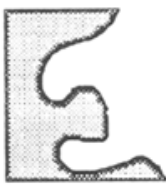

CA

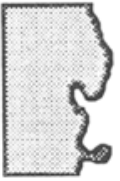

DA

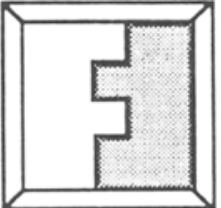

Figure A.

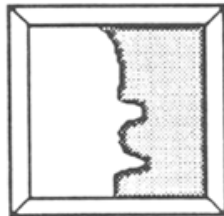

Figure B

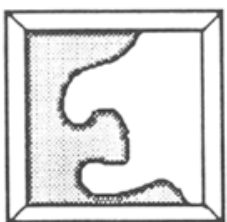

Figure C.

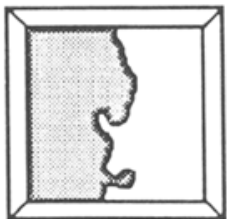

Figure D.

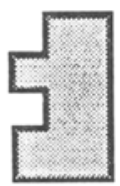

$A B$

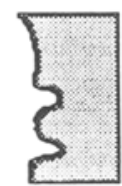

$B B$

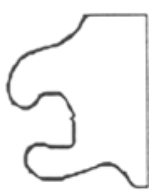

CB

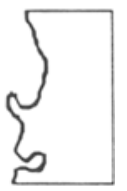

DB
Figure 4. Ambiguous and inspection figures for Experiment 2.

line around one side of an unambiguous figure and indicating the form that the other half had when it was seen as a "ingure." They were told that both the figures presented and those imagined would be on a recognition test given at the end of the experiment.

The distractor and inspection figures (shown in Figures 2 and 4 , respectively) were presented for $10 \mathrm{sec}$, and the ambiguous figures were presented for $30 \mathrm{sec}$. A 30-sec test interval was selected, because in Experiment 1 a number of subjects had not reversed the figures after $10 \mathrm{sec}$. The trial series for the Familiar, Imagery/ Unfamiliar, and Unfamiliar groups was as follows: Distractor 1, Distractor 2, Inspection 1, Reversible Figure 1, Distractor 3, Inspection 2, Reversible Figure 2, Distractor 4, Distractor 5, Inspection Figure 3, Reversible Figure 3, Distractor 6, Inspection 4, Reversible Figure 4 . The same order was used for the No-Inspection group (although no inspection figures were presented) and for Group A/B (both inspection figures were presented during the inspection phase). The inspection/figure-ground pairs were presented randomly, and the distractor figures were presented in the same order.

There were two subgroups per group, with half of the subjects in each. The subjects in Subgroup 1 saw Inspection Figures AB, BA, CA, and DB, whereas those in Subgroup 2 saw AA, BB, CB, and DA (see Figure 4). The subgroup figures were selected so that each had two left and two right inspection figures and one from each pair was gray and one was white. The subjects from Group A/B were also placed in a subgroup. In this case, the subgroup designated which inspection figure immediately preceded the ambiguous test figure. Again, subgroup was not a factor of interest, but simply served as an internal control.

\section{Results}

The data were analyzed according to the measures employed in Experiment 1, and they are defined the same way. Summary data are presented in Table 4.

\section{Control Data}

In order to identify any preferences for seeing the right versus the left form as figure, the mean duration across the four test figures was calculated. The mean duration for the left side was $16 \mathrm{sec}$, and the mean for the right was $14 \sec [t(15)=1.31, p>.10]$. Thus, the left- and right-side versions were seen for approximately the same amount of time. The initial perception data were also tallied. Collapsed across the figures, the left side was seen as a figure first $46 \%$ of the time, and the right side was seen as a figure first $54 \%$ of the time $[t(15)=.522, p>$ $.10]$. Other aspects of the control group data will be reported within the context of specific experimental questions and statistical tests.

\section{Number of Reversals}

It was predicted that groups Familiar, A/B, and Imagery/Unfamiliar would report a greater number of reversals in the 30-sec test phase than would the No-Inspection

Table 3

Treatment of the Groups in Experiment 2

\begin{tabular}{|c|c|c|c|c|c|}
\hline \multirow[b]{2}{*}{ Phase } & \multicolumn{5}{|c|}{ Group } \\
\hline & No-Inspection & Unfamiliar & Familiar & A/B & Imagery/Unfamiliar \\
\hline \multicolumn{6}{|l|}{ Learning } \\
\hline Figures & target & nontarget & target & target & nontarget \\
\hline \multicolumn{6}{|l|}{ Practice } \\
\hline Figures & nontarget & nontarget & nontarget & nontarget & nontarget \\
\hline \multicolumn{6}{|l|}{ Experimental } \\
\hline Instructions & $\begin{array}{l}\text { learn figures, } \\
\text { report reversals }\end{array}$ & $\begin{array}{l}\text { learn figures, } \\
\text { report reversals }\end{array}$ & $\begin{array}{l}\text { learn figures, } \\
\text { report reversals }\end{array}$ & $\begin{array}{l}\text { learn figures, } \\
\text { report reversals }\end{array}$ & $\begin{array}{l}\text { learn and image figures, } \\
\text { report reversals }\end{array}$ \\
\hline $\begin{array}{l}\text { Test figures } \\
\text { Inspection figures }\end{array}$ & $\begin{array}{l}\text { target } \\
\text { none }\end{array}$ & $\begin{array}{l}\text { target } \\
\text { one }\end{array}$ & $\begin{array}{l}\text { target } \\
\text { one }\end{array}$ & $\begin{array}{l}\text { target } \\
\text { both }\end{array}$ & $\begin{array}{l}\text { target } \\
\text { see one and imagine the }\end{array}$ \\
\hline
\end{tabular}


Table 4

Summary Data From Experiment 2

\begin{tabular}{lccccc} 
& \multicolumn{5}{c}{ Group } \\
\cline { 2 - 6 } \multicolumn{1}{c}{ Dependent Variable } & No-Inspection & Unfamiliar & Familiar & A/B & $\begin{array}{c}\text { Imagery/ } \\
\text { Unfamiliar }\end{array}$ \\
\hline Mean No. of reversals & 5.31 & 6.50 & 13.11 & 14.02 & 15.20 \\
Duration (same, in seconds) & & 15.65 & 15.94 & 15.24 & 15.52 \\
Initial percept (different) & & $41 \%$ & $47 \%$ & $58 \%$ & $59 \%$ \\
\hline
\end{tabular}

and the Unfamiliar groups. Further, it was predicted that the Imagery/Unfamiliar and A/B groups would have a comparable number of reversals, both of which would be higher than that for the Familiar group. This prediction was based on the following reasoning: The Familiar group had the potential to undergo a deep level of processing during the inspection phase through the activation of the memory of the alternate version. We have proposed that this activation can lead to the increased perceived ambiguity of the inspection stimulus such that a "reversal potential" develops while the figure is viewed. Group A/B had specific immediate past experience with both alternatives; therefore, both alternatives were available in working memory at the time that the ambiguous figure was presented. This condition was created to maximize the state of availability of both versions of the figure, an availability that may not have been consistently present for the subjects in the Familiar group. It is easy to imagine that availability would vary across subjects in the Familiar group because of individual differences in processing strategies. In some sense, Condition A/B was creating a state of affairs that we claim is similar to the one in the Familiar group but more consistently across subjects. The Imagery/Unfamiliar condition presented an interesting variation on the availability concept. These subjects were not given specific past experience with the target figures prior to the test, yet they were actively engaged in imagining the alternative on line. This should have created a condition similar to the $A / B$ condition.

It was predicted that the No-Inspection and Unfamiliar groups would report a comparable number of reversals; although the experimental treatments were different, both treatments were aimed at creating a shallow level of processing. In the case of the No-Inspection subjects, there was no inspection figure and thus no opportunity for the perception of the ambiguous figure to be changed by the processing that would occur during this stage. The only information to be brought to bear would have been the exposure to the figures during the learning phase. This information could be applied once the ambiguous figures were presented.

The model to be tested can be represented as the following with the contrast coefficients used in parentheses:

$$
\text { No-Inspection } \begin{aligned}
(-1.5) & =\operatorname{Unfamiliar}(-1.5) \\
& <\text { Familiar }(-1.0)<\mathrm{A} / \mathrm{B}(2.0) \\
& =\text { Imagery/Unfamiliar }(2.0) .
\end{aligned}
$$

This analysis was significant $[F(1,75)=7.90, p=.006]$.
Of particular interest was the difference in reversal number between the Imagery/Unfamiliar and Unfamiliar groups $[t(30)=2.22, p<.03]$, since these groups received the same preexperimental treatment and received the same perceptual stimulus during the inspection phase. Clearly, the active process of imaging the alternative while seeing the version that was given perceptually created an increased state of perceived ambiguity.

\section{Duration}

This analysis was based on the mean duration for the inspected version across the four target figures. The NoInspection group was not included in this analysis because those subjects did not receive the inspection figures. The model can be stated as follows with the contrast coefficients given in parentheses:

$$
\begin{aligned}
\text { Unfamiliar }(-2.0) & <\text { Familiar }(-1.0)<\mathrm{A} / \mathrm{B}(1.5) \\
& =\text { Imagery/Unfamiliar }(1.5) .
\end{aligned}
$$

This test yielded $F(1,41)=.19, p>.10$. Again, there was no difference among the means.

\section{Initial Perception Data}

This variable was defined as whether the first perception of the ambiguous figure was the same as the inspection form, or whether it was different (the alternate version). The percent of different responses for the four initial percepts was the dependent measure. The specific figure effects were not examined. The predictions were based on the reasoning above. The model can be stated as follows with the contrast coefficients given in parentheses:

$$
\begin{aligned}
\text { Unfamiliar }(-2.0) & <\text { Familiar }(-1.0)<\mathrm{A} / \mathrm{B}(1.5) \\
& =\text { Imagery/Unfamiliar }(1.5) .
\end{aligned}
$$

This test yielded significance $[F(1,60)=6.71, p=.012]$.

\section{Discussion}

The findings of Experiment 2 failed to support a neural fatigue model of reversal. It was hypothesized, and largely confirmed, that as the amount and availability of information regarding the alternate percept increased during the inspection phase, so did the perceptual instability of the figure. This effect emerged in connection with the initial perception and reversal data. As was suggested in the introduction, it could well be that the prolongedinspection technique does not create a situation in which satiation accumulates, but rather one in which the alter- 
nate percept becomes available, through processes involving memory or imagination.

Examining the information available to a subject is informative. The No-Inspection (control) group received exposure to the target figures during the learning phase but did not receive an inspection figure during the experimental phase. These subjects exhibited the lowest number of reversals (5.31). The Unfamiliar group obtained a similar reversal number (6.50), even though this group received the inspection phase. The juxtaposition of these two groups points to the importance of both the preexperimental exposure to the target figures and the essential role of the inspection phase as a period in which "reversal potential" is created and in which a general processing shift may occur. If the critical determinant were exposure to the target figures during the learning phase, one would predict that the No-Inspection group would have obtained a greater reversal number than would the Unfamiliar group, and furthermore, that the reversal number would be similar to that obtained in the Familiar group. That was not the case. The Familiar group had a significantly larger number of reversals (13.11). The Imagery/Unfamiliar subjects were not exposed to the target figures during the learning phase, but they were given explicit instructions to discover the alternate version of the inspection figures by imagining what they would look like. A comparison of the initial perception data in the Unfamiliar and Imagery/Unfamiliar groups revealed that subjects in the latter group were more likely to report the different percept than were those in the former group. In addition, the Imagery/Unfamiliar group obtained a significantly greater number of reversals (15.20). The imagery instructions compensated for the lack of perceptual experience with the figures in the learning phase. It is interesting to note that imaging the alternate figure exerts the same influence as does having both versions available. Group A/B was exposed to both interpretations of the ambiguous figure during the inspection phase, thereby making these representations available in short-term memory. The Imagery/Unfamiliar group inspected one version and imagined the other, which also made both representations available in short-term memory. These groups had a comparable number of reversals and the largest proportion of different initial perception responses. Both imaging and remembering are sources of information that render the alternate percept available in short-term memory. What is particularly compelling here is that the subjects in Group A/B were informed and received both inspection figures during the inspection phase, whereas the subjects in the Imagery/Unfamiliar condition were not informed and received only one of the inspection stimuli. Despite this difference, the results from the two groups are virtually identical. This suggests that imagined experience is just as potent in this context as experience obtained via externally derived information.

Finke $(1980,1986)$ has discussed in detail the functional similarity between images and percepts, demonstrating that in some instances both lead to the same behavioral consequences. For example, Finke and Schmidt (1977) demonstrated that the McCollough effect can be induced through imagery. When imagination instructions replaced the color or bar patterns (given the condition), the typical effect, although weaker than that obtained in the perception condition, resulted. Bagnara, Simion, Tagliabue, and Umiltà (1988) have demonstrated that visual images are often generated rapidly and automatically under certain conditions. Neisser (1976), in a discussion of perceptual set, stated, "To have a perceptual set for something is to have an image. The more precisely that image anticipates the information to come, the more effective the set should be" (p. 145). In the terms of Warren (1985), perception is an active evaluative process that establishes "criterion shift rules" to ensure that perception "remains appropriate to current conditions"' (p. 574). Hochberg (1970) stated that perceptual reversal is a function of selective attention in which the "selection is between two maps or sets of expectations" (p. 120).

In the present experiment, all the subjects were "set" to see reversible figures, but in the Imagery/Unfamiliar, A/B, and Familiar groups, the set provided by the inspection phase anticipated the information to come. That is, the information to come was ambiguous and there were two interpretations that could arise from this input. The set, as conceptualized here, was effective in terms of providing the visual system with flexibility of interpretation.

\section{A REINTERPRETATION OF THE SATIATION STUDIES}

It could be that subjects in a classic "satiation" study used the memory of the noninspected version to image its presence during the inspection phase, thus bringing about a perceptual state of affairs that appeared to be a result of satiation. Subjects in all of these studies had repeated exposure to the target ambiguous figures as well as repeated exposure to both inspection versions of the figures. This design would increase the strength and availability of the memories of both versions, leading to retrieval of the memory of the alternate version during the inspection period. It would also increase the likelihood that subjects could experience the memory as image, and see intimations of the noninspected versions in the inspection stimuli. The within-subject repeated measures design employed in these studies produced results that upon first glance appeared to support satiation.

Why does the uninspected alternative, once made available, become "preferred" or sought when the ambiguous figure is presented? A preference for novelty may be a fundamental human characteristic. Fantz and others have demonstrated attraction to novelty in infants (Fantz, 1964; Slater, Morison, Rose, 1983; Weizmann, Cohen, \& Pratt, 1971). Habituation is created when an infant is familiarized with a stimulus. When presented with both the familiar and a novel stimulus together, infants will fixate the novel object longer than the familiar one. A retinaladaptation model of habituation has been ruled out (Slater 
et al., 1983). The exact locus of the habituation effect is unclear, although Fantz (1964) has described it thus: "The present results indicate perception, recognition, and satiation of interest of a particular pattern. This effect of specific previous experiences is 'learning' in the broad meaning of the term" (p. 669). Similarly, "novel popout" of unfamiliar word stimuli has recently been demonstrated after many trials with both familiar and novel stimuli (Johnston, Hawley, Plewe, Elliott, \& DeWitt, 1990). In the satiation studies, the choice was between one of two interpretations of an ambiguous stimulus, one of which was certainly more familiar or habituated.

Thus, we presume that something akin to a "satiation of interest" may indeed account for previous results, but that this is not a process that is color-specific-as argued by Hochberg (1950) - or location-specific - as argued by Toppino and Long (1987) - or that requires lengthy fatiguing of neural channels. Rather, the organism "seeks" or is attracted by novelty, a process that may be automatic (Johnston et al., 1990) and that may have adaptive significance.

The experiments reported in this paper were conducted with the intention of providing support for an alternative explanation of figural reversals and to arrive at a better understanding of how memories and imagination affect the perception of ambiguous stimuli. Experiment 1 identified the critical role of focal attention to the inspection figures. This result is contrary to what would be predicted by a satiation hypothesis, in which little or no attention to the stimulus ought to be required. Attention, paired with memories of the alternatives, provided a condition that yielded a greater number of reversals as compared to a group that received the same preexperimental treatment, but did not provide focal attention to the inspection figures. It was also found that focal attention did not bring about the same result when subjects were aware of ambiguity but did not have experience with the target test figures. Results from the Attention/Unfamiliar condition were similar to those from the Inattention/Familiar group. These results designated the inspection phase as one that initiates learning and not satiation.

The learning that occurs is apparently a function of the interaction of the inspection stimulus and the memory of the alternative. It could also be that the learning occurs as a result of a process in which subjects discover the missing figure that would be paired with the given inspection form. We proposed that the interaction between the inspection figure and memory would be greatest when this synthesis led to a state in which the as-yet-unseen version would take on a perceptual strength, which in turn would influence the perception of the ambiguous figure. The intimation of the version not given in the inspection figure might take on the character of an "unfinished percept."

- The importance of the process of imaging was examined in Experiment 2. Even though these subjects did not have exposure to the alternatives of the reversible figureground patterns prior to the test, they showed the greatest degree of perceptual change when presented with the am- biguous figure. It would be interesting to test this variable further, in conjunction with memories formed prior to the experiment. This would require the use of informed subjects as in the Familiar group, with the additional instructions to image the missing figure while viewing the inspection stimulus. Our goal in the present experiment was simply to examine the effectiveness of the imaging variable alone with the assumption that a memory would be created "on line" as a function of the imaging. As was learned from the results, this imaging variable seems to be very effective in initiating a change.

When one speaks of the effect of intention on perception, it is possible that the realization of the intended state comes forth through the evocation of a visual image of what is intended. According to Hochberg (1970), "reversal occurs when active looking is relaxed and attention falters" (p. 120). We contend that reversal occurs as the end result of a process that occurs during active looking. For us, active looking implies the intervention of memories and/or imagination.

\section{REFERENCES}

Babich, S., \& Standing, L. (1981). Satiation effects with reversible figures. Perceptual \& Motor Skills, 52, 203-210.

Bagnara, S., Simion, F., Tagliabue, M. E., \& Umiltà, C. (1988). Comparison processes on visual mental images. Memory \& Cognition, 16, 138-146.

CARLSON, V. R. (1953). Satiation in a reversible perspective figure. Journal of Experimental Psychology, 45, 442-448.

COHEN, L. (1959). Rate of apparent change of a Necker cube as a function of prior stimulation. American Journal of Psychology, 72, 327-344.

CoRNwell, H. G. (1963). Prior experience as a determinant of figureground organization. Joumal of Experimental Psychology, 65, 156-162.

CORNWELL, H. G. (1964). Effect of training on figure-ground organization. Journal of Experimental Psychology, 68, 108-109.

EPSTEIN, W., \& Rock, I. (1960). Perceptual set as an artifact of recency. American Joumal of Psychology, 73, 214-228.

FANTZ, R. L. (1964). Visual experience in infants: Decreased attention to familiar patterns relative to novel ones. Science, 146, 668-670.

FINKE, R. (1980). Levels of equivalence in imagery and perception. Psychological Review, 87, 113-132.

FINKE, R. (1986). Mental imagery and the visual system. Scientific American, 253, 88-95.

FINKE, R., \& SCHMIDT, M. (1977). Orientation-specific color aftereffects following imagination. Joumal of Experimental Psychology: Human Perception \& Performance, 3, 599-606.

FluegaL, J. C. (1912). The influence of attention in illusions of reversible perspective. British Journal of Psychology, 5, 357-416.

GiRGUS, J. J., Rock, I., \& EGATZ, R. (1977). The effect of knowledge of reversibility on the reversibility of ambiguous figures. Perception \& Psychophysics, 22, 550-556.

HOCHBERG, J. (1950). Figure-ground reversal as a function of visual satiation. Joumal of Experimental Psychology, 40, 682-686.

HochBERG, J. (1970). Attention, organization, and consciousness. In D. I. Mostofsky (Ed.), Attention: Contemporary theory and analysis (pp. 99-124). New York: Appleton-Century-Crofts.

HoRLITZ, K. L. (1988, April). Satiation versus past experience in the perception of reversible figures. In Proceedings of the 59th Annual Meeting of the Easterm Psychological Association (p. 40), Buffalo, NY. (Abstract)

HoRLITZ, K. L. (1990). Figural reversals reappraised: The effects of attention, memory and imagery on the perception of ambiguous figures. Unpublished doctoral dissertation, Rutgers, The State University of New Jersey, New Brunswick.

Johnston, W. A., Hawley, K. J., Plewe, S. H., Elliott, J. M. G., 
\& DEWITT, M. J. (1990). Attention captured by novel stimuli. Journal of Experimental Psychology: General, 119, 397-411.

KOEHLER, W. (1940). Dynamics in psychology. New York: Liveright. LEEPER, R. (1935). A study of a neglected portion of the field of learning-the development of sensory organization. Journal of Genetic Psychology, 46, 41-75.

LieBert, R. M., \&URK, B. (1985). Voluntary control of reversible figures. Perceptual \& Motor Skills, 61, 1307-1310.

NeIsSer, U. (1976). Cognition and reality. San Francisco: W. H. Freeman.

Olson, R., \& ORBaCh, J. (1966). Reversibility of the Necker cube: VIII. Parts of the figure contributing to the perception of reversals. Perceptual \& Motor Skills, 22, 623-629.

Orbach, J., Ehrlich, D., \& Heath, A. A. (1963). Reversibility of the Necker cube: 1 . An examination of the concept of "satiation of orientation." Perceptual \& Motor Skills, 17, 439-458.

Orbach, J., Ehrlich, D., \& Vainstein, E. (1963). Reversibilty of the Necker cube: III. Effects of interpolation on reversal rate of the cube presented repetitively. Perceptual \& Motor Skills, 17, 571-582.

Peterson, M. A.(1986). Illusory concomitant motion in ambiguous stereograms: Evidence for nonstimulus contributions to perceptual organization. Journal of Experimental Psychology: Human Perception \& Performance, 12, 50-60.

Peterson, M. A., Hochberg, J. (1983). Opposed-set measurement procedure: A quantitative analysis of the role of local cues and intention in form perception. Journal of Experimental Psychology: Human Perception \& Performance, 9, 183-193.

Peterson, M. A., \& Hochberg, J. (1989). Necessary considerations for a theory of form perception: A theoretical and empirical reply to Boselie and Leewenberg (1986). Perception, 18, 105-119.

Reisberg, D., \& O'Shaughnessy, M. (1984). Diverting subjects' concentration slows figural reversals. Perception, 13, 461-468.

RoCK, I., \& GUTMAN, D. (1981). The effect of inattention on form perception. Journal of Experimental Psychology: Human Perception \& Performance, 7, 275-285.

RoCK, I., \& MitchNer, K. (1992). Further evidence of failure of reversal of ambiguous figures by uninformed subjects. Perception, 21, 39-45.

Rosenthal, R., \& Rosnow, R. L. (1985). Contrast analysis: Focused comparisons in the analysis of variance. New York: Cambridge University Press.

Slater, A., Morison, V., \& Rose, D. (1983). Locus of habituation in the human newborn. Perception, 12, 593-598.

SpITZ, H. H., \& Lipman, R. S. (1962). Some factors affecting Necker cube reversal rate. Perceptual \& Motor Skills, 15, 611-625.

ToPPINO, T. C., \& LoNG, G. M. (1987). Selective adaptation with reversible figures: Don't change that channel. Perception \& Psychophysics, 42, 37-48.

TSAL, Y., \& KolberT, L. (1985). Disambiguating ambiguous figures by selective attention. Quarterly Journal of Experimental Psychology, 37A, 25-37.

VETTER, R. J. (1965). Perception of ambiguous figure-ground patterns as a function of past experience. Perceptual \& Motor Skills, 20, 183-188.

VIRsu, V. (1975). Determination of perspective reversals. Nature, 257, 786-787.

von Grunau, M. W., Wiggin, S., \& Reed, M. (1984). The local character of perspective organization. Perception \& Psychophysics, 35, 319-324.

WARREN, R. M. (1985). Criterion shift rule and perceptual homeostasis. Psychological Review, 92, 574-584.

Weizmann, F., Cohen, L., \& Pratt, R. J. (1971). Novelty, familiarity, and the development of infant attention. Developmental Psychology, 4, 149-154.

\section{NOTES}

1. Additional studies that support a past experience effect using a different method (Cornwell, 1963, 1964; Vetter, 1965) showed that exposure to biased figure-ground patterns during a learning phase influenced a subject's initial perception of these figures when the unbiased counterparts were presented again during a test phase.

2. This response method was selected because it reduced interference while attending to the figures. It was confirmed to be a sensitive measure of reversals, which was supported by the replication of reversal results in Experiment 2.

3. Rock and Gutman (1981) presented overlapping figures (one in red and one in green) for $1 \mathrm{sec}$, providing subjects with instructions to attend, for instance, to the red figures. After a number of figures were presented in this manner, the subjects were given a recognition test. Very few of the unattended figures were recognized. According to Rock and Gutman, the lack of memory for the unattended items suggested that these items were not perceived. In the present experiment, the figures used were not overlapped with another figure and they were presented for $60 \mathrm{sec}$ instead of $1 \mathrm{sec}$. This, paired with the postexperimental interviews, suggests that subjects could perceive the figure but that the level of processing was shallow.

4. A 10-sec inspection phase was deemed of sufficient duration on the basis of the results of a preliminary study that indicated no significant differences between a 10 -sec inspection phase and a 60 -sec one for any of the dependent measures used in the present study (Horlitz, 1990, Experiment 2). In fact, this finding may itself be taken as evidence against the satiation hypothesis; duration of exposure should affect satiation, but it is less likely to affect the ability of "higher" cognitive processes to produce an apprehension of the alternate percept.

(Manuscript received January 29, 1991; revision accepted for publication October $24,1992$. 